\title{
Noncommutative-Geometry Inspired Charged Wormholes with Low Tidal Forces
}

\author{
Peter K. F. Kuhfittig, Vance D. Gladney \\ Department of Mathematics, Milwaukee School of Engineering, Milwaukee, WI, USA \\ Email:kuhfitti@msoe.edu
}

How to cite this paper: Kuhfittig, P.K.F. and Gladney, V.D. (2017) NoncommutativeGeometry Inspired Charged Wormholes with Low Tidal Forces. Journal of Applied Mathematics and Physics, 5, 574-581. https://doi.org/10.4236/jamp.2017.53049

Received: January 22, 2017

Accepted: March 5, 2017

Published: March 8, 2017

Copyright $\odot 2017$ by authors and Scientific Research Publishing Inc. This work is licensed under the Creative Commons Attribution International License (CC BY 4.0).

http://creativecommons.org/licenses/by/4.0/

(c) (i) Open Access

\begin{abstract}
When Morris and Thorne first proposed that wormholes might be actual physical structures suitable for interstellar travel, they needed to pay close attention to certain traversability conditions such as low tidal forces, which placed severe constraints on the wormhole geometry. Even more problematical was the need for "exotic matter" resulting from the unavoidable violation of the null energy condition required to hold a wormhole open. The purpose of this paper is to overcome these problems by starting with the charged wormhole model of Kim and Lee and assuming a noncommutative-geometry background: the violation of the null energy condition can be attributed to the latter, while the electric charge allows the reduction of the tidal forces to acceptable levels without invoking the trivial zero-tidal-force assumption.
\end{abstract}

\section{Keywords}

Charged Wormholes, Noncommutative Geometry, Tidal Constraints

\section{Introduction}

Wormholes are handles or tunnels in spacetime that link widely separated regions of our Universe or different universes altogether. Morris and Thorne [1] proposed the following line element for the wormhole spacetime:

$$
\mathrm{d} s^{2}=-\mathrm{e}^{2 \Phi(r)} \mathrm{d} t^{2}+\frac{\mathrm{d} r^{2}}{1-b(r) / r}+r^{2}\left(\mathrm{~d} \theta^{2}+\sin ^{2} \theta \mathrm{d} \phi^{2}\right),
$$

using units in which $c=G=1$. In this line element, $b=b(r)$ is called the shape function and $\Phi=\Phi(r)$ is called the redshift function, which must be everywhere finite to avoid an event horizon. For the shape function we must have $b\left(r_{0}\right)=r_{0}$, where $r=r_{0}$ is the radius of the throat of the wormhole. An important requirement is the flare-out condition at the throat: $b^{\prime}\left(r_{0}\right)<1$, while $b(r)<r$ near the throat. The flare-out condition can only be met by violating 
the null energy condition

$$
T_{\alpha \beta} k^{\alpha} k^{\beta} \geq 0
$$

for all null vectors $k^{\alpha}$, where $T_{\alpha \beta}$ is the stress-energy tensor. Matter that violates this condition is referred to as "exotic" in Ref. [1]. In particular, for the radial outgoing null vector $(1,1,0,0)$, the violation reads $T_{\alpha \beta} k^{\alpha} k^{\beta}=\rho+p_{r}<0$. Here $T_{t}^{t}=-\rho(r)$, the energy density, and $T_{r}^{r}=p_{r}(r)$, the radial pressure. $\left(T_{\theta}^{\theta}=T_{\phi}^{\phi}=p_{t}(r)\right.$, the lateral pressure.)

In Ref. [1], Morris and Thorne also discussed the tidal forces that a traveler would be subjected to and subsequently put the following constraint on the redshift function: $\Phi^{\prime}(r) \leq g_{\oplus} /\left(c^{2} \sqrt{1-b(r) / r}\right)$. The feasibility of this assumption will be discussed later.

The purpose of this paper is to revisit these requirements by starting with the modified charged wormhole model due to Kim and Lee [2] and assuming a noncommutative-geometry background.

Similar issues involving traversable wormholes in Lovelock gravity are discussed in Refs. [3] [4] [5], with emphasis on the energy conditions. In particular, Ref. [5] considers higher-dimensional thin-shell wormholes. Einstein-GaussBonnet wormholes satisfying the weak energy condition are studied in Ref. [6], while higher-dimensional evolving wormholes satisfying the null energy condition are discussed in Ref. [7]. Regarding the tidal constraints, it was noted in Ref. [8] that the zero-tidal-force assumption is incompatible with quantum field theory in classical general relativity.

\section{Noncommutative Geometry}

An important outcome of string theory is the realization that coordinates may become noncommutative operators on a $D$-brane [9] [10]. The result is a fundamental discretization of spacetime due to the commutator $\left[\boldsymbol{x}^{\mu}, \boldsymbol{x}^{\nu}\right]=i \theta^{\mu \nu}$, where $\theta^{\mu \nu}$ is an antisymmetric matrix. According to Refs. [11] [12] [13], noncommutativity replaces point-like objects by smeared objects, thereby eliminating the divergences that normally appear in general relativity.

Applications of noncommutative geometry are numerous and varied. One of the first was to wormholes in semi-classical gravity by Garattini and Lobo [14]. The same authors analyzed the stability of gravastars [15]. Gravitational lensing of wormholes in noncommutative geometry was studied in Ref. [16], while the search for higher-dimensional wormholes was proposed in Ref. [17].

It seems natural to model the smearing by means of a Gaussian distribution of minimal length $\sqrt{\alpha}$ instead of the Dirac delta function [12] [13] [17] [18]. A simpler but equally effective way is to assume that the energy density of the static and spherically symmetric and particle-like gravitational source has the form

$$
\rho(r)=\frac{M \sqrt{\alpha}}{\pi^{2}\left(r^{2}+\alpha\right)^{2}} ;
$$

(see Refs. [19] and [20]). The point is that the mass $M$ is diffused throughout 
the region of linear dimension $\sqrt{\alpha}$ due to the uncertainty. It should be noted that noncommutative geometry is an intrinsic property of spacetime and does not depend on particular features such as curvature.

Next, we note that the Einstein field equations $G_{\hat{\mu} \hat{v}}=8 \pi T_{\hat{\mu} \hat{v}}$ result in the following forms:

$$
\begin{gathered}
\rho(r)=\frac{b^{\prime}}{8 \pi r^{2}}, \\
p_{r}(r)=\frac{1}{8 \pi}\left[-\frac{b}{r^{3}}+2\left(1-\frac{b}{r}\right) \frac{\Phi^{\prime}}{r}\right],
\end{gathered}
$$

and

$$
p_{t}(r)=\frac{1}{8 \pi}\left(1-\frac{b}{r}\right)\left[\Phi^{\prime \prime}-\frac{b^{\prime} r-b}{2 r(r-b)} \Phi^{\prime}+\left(\Phi^{\prime}\right)^{2}+\frac{\Phi^{\prime}}{r}-\frac{b^{\prime} r-b}{2 r^{2}(r-b)}\right] .
$$

Here $p_{r}(r)$ is the radial pressure and $p_{t}(r)$ is the lateral pressure. The conservation law $T_{; \nu}^{\mu \nu}=0$ implies that only two of Equations (4)-(6) are independent. So Equation (6) can be obtained from the other two. (See Ref. [21] for further details.)

As a final comment, to make use of Equation (3), we can keep the standard form of the Einstein field equations in the sense that the Einstein tensor retains its original form, but the stress-energy tensor is modified [12]. It follows that the length scale used need not be restricted to the Planck scale.

\section{The Modified Kim-Lee Model}

Since black holes can carry an electric charge, it is natural to assume that wormholes can do likewise. So it was proposed by Kim and Lee [2] that for a wormhole with constant electric charge $Q$, the Einstein field equations take on the form

$$
G_{\mu \nu}^{(0)}+G_{\mu \nu}^{(1)}=8 \pi\left[T_{\mu \nu}^{(0)}+T_{\mu \nu}^{(1)}\right] .
$$

Given that the usual form is $G_{\mu \nu}^{(0)}=8 \pi T_{\mu \nu}^{(0)}$, the modified form in Equation (7) is obtained by adding the matter term $T_{\mu \nu}^{(1)}$ to the right side and the corresponding back reaction term $G_{\mu \nu}^{(1)}$ to the left side. The proposed metric is

$$
\mathrm{ds} s^{2}=-\left(1+\frac{Q^{2}}{r^{2}}\right) \mathrm{d} t^{2}+\left(1-\frac{b(r)}{r}+\frac{Q^{2}}{r^{2}}\right)^{-1} \mathrm{~d} r^{2}+r^{2}\left(\mathrm{~d} \theta^{2}+\sin ^{2} \theta \mathrm{d} \phi^{2}\right) .
$$

Observe that whenever $b \equiv 0$, the wormhole becomes a Reissner-Nordström black hole, and if $Q=0$, the spacetime reverts to a Morris-Thorne wormhole, where the shape function $b=b(r)$ meets the usual requirements. Kim and Lee go on to show that the metric, Equation (8), is a self-consistent solution of the Einstein field equations. The shape function $b=b(r)$ of the Morris-Thorne wormhole is now replaced by the effective shape function

$$
b_{\text {eff }}(r)=b(r)-\frac{Q^{2}}{r},
$$

to be discussed later. 
To overcome certain difficulties in determining the tidal constraints, we will use the following modified metric already introduced in Refs. [22] and [23]:

$$
\mathrm{ds}^{2}=-\left(1+S(r)+\frac{Q^{2}}{r^{2}}\right) \mathrm{d} t^{2}+\left(1-\frac{b(r)}{r}+\frac{Q^{2}}{r^{2}}\right)^{-1} \mathrm{~d} r^{2}+r^{2}\left(\mathrm{~d} \theta^{2}+\sin ^{2} \theta \mathrm{d} \phi^{2}\right) .
$$

here $S(r)$ is a differentiable function such that $S(r)>0$ and $S^{\prime}(r)>0$. As noted in Ref. [22], line element (10) remains a valid solution of the Einstein field equations.

\section{The Modified Kim-Lee Model in Noncommutative Geometry}

In the Kim-Lee model, Equation (8), and in the modified model, Equation (10),

$$
\text { total charge }=\iiint_{V} \rho_{q}(r) \mathrm{d} V,
$$

where $\rho_{q}(r)$ is the charge density. Adapting this to the noncommutative-geometry background, we assume that the charge density has the form

$$
\rho_{q}(r)=\frac{Q^{2} \sqrt{\alpha}}{\pi^{2}\left(r^{2}+\alpha\right)^{2}},
$$

where $Q^{2}$ refers to the Kim-Lee model. To obtain the smeared charge $Q_{\alpha}^{2}(r)$, we evaluate

$$
Q_{\alpha}^{2}(r)=\int_{r_{0}}^{r} 4 \pi\left(r^{\prime}\right)^{2} \frac{Q^{2} \sqrt{\alpha}}{\pi^{2}\left(r^{2}+\alpha\right)^{2}} \mathrm{~d} r^{\prime}=\frac{2 Q^{2} \sqrt{\alpha}}{\pi}\left(\frac{1}{\sqrt{\alpha}} \tan ^{-1} \frac{r}{\sqrt{\alpha}}-\frac{r}{r^{2}+\alpha}\right) .
$$

Observe that $Q_{\alpha}^{2}\left(r_{0}\right)=0$; also, seen from a large distance, $Q_{\alpha}^{2}(r)$ becomes $Q^{2}$.

Similarly, from Equation (4) and Equation (9), we get for the effective shape function,

$b_{\text {eff }}(r)$

$=\frac{4 M \sqrt{\alpha}}{\pi}\left(\frac{1}{\sqrt{\alpha}} \tan ^{-1} \frac{r}{\sqrt{\alpha}}-\frac{r}{r^{2}+\alpha}\right)-\frac{1}{r} \frac{2 Q^{2} \sqrt{\alpha}}{\pi}\left(\frac{1}{\sqrt{\alpha}} \tan ^{-1} \frac{r}{\sqrt{\alpha}}-\frac{r}{r^{2}+\alpha}\right)+C$.

Since $Q_{\alpha}^{2}\left(r_{0}\right)=0$, we obtain from $b\left(r_{0}\right)=r_{0}$,

$$
b_{\text {eff }}\left(r_{0}\right)=\frac{4 M \sqrt{\alpha}}{\pi}\left(\frac{1}{\sqrt{\alpha}} \tan ^{-1} \frac{r_{0}}{\sqrt{\alpha}}-\frac{r_{0}}{r_{0}^{2}+\alpha}\right)-0+C=r_{0}
$$

and hence the constant $C$. Thus

$$
\begin{aligned}
b_{\text {eff }}(r)= & \frac{4 M \sqrt{\alpha}}{\pi}\left(\frac{1}{\sqrt{\alpha}} \tan ^{-1} \frac{r}{\sqrt{\alpha}}-\frac{r}{r^{2}+\alpha}\right)-\frac{1}{r} \frac{2 Q^{2} \sqrt{\alpha}}{\pi}\left(\frac{1}{\sqrt{\alpha}} \tan ^{-1} \frac{r}{\sqrt{\alpha}}-\frac{r}{r^{2}+\alpha}\right) \\
& -\frac{4 M \sqrt{\alpha}}{\pi}\left(\frac{1}{\sqrt{\alpha}} \tan ^{-1} \frac{r_{0}}{\sqrt{\alpha}}-\frac{r_{0}}{r_{0}^{2}+\alpha}\right)+r_{0} .
\end{aligned}
$$

For a wormhole solution, we require that $b_{\text {eff }}^{\prime}\left(r_{0}\right)>0$ and $b_{\text {eff }}^{\prime}\left(r_{0}\right)<1$, the flare-out condition mentioned earlier. To that end, we determine

$b_{\text {eff }}^{\prime}\left(r_{0}\right)=\frac{4 M \sqrt{\alpha}}{\pi} \frac{2 r_{0}^{2}}{\left(r_{0}^{2}+\alpha\right)^{2}}-\frac{1}{r_{0}} \frac{2 Q^{2} \sqrt{\alpha}}{\pi} \frac{2 r_{0}^{2}}{\left(r_{0}^{2}+\alpha\right)^{2}}=\frac{4 r_{0}^{2} \sqrt{\alpha}}{\pi\left(r_{0}^{2}+\alpha\right)^{2}}\left(2 M-\frac{1}{r_{0}} Q^{2}\right)$ 
since $Q_{\alpha}^{2}\left(r_{0}\right)=0$. Looking ahead to the traversability conditions which involve measurements in meters, we will assume that $r_{0}>1 \mathrm{~m}$. So to keep $b_{\text {eff }}^{\prime}\left(r_{0}\right)$ positive, we must have

$$
Q^{2} \leq 2 M
$$

Since $\sqrt{\alpha} \ll M$, it also follows that

$$
b_{\text {eff }}^{\prime}\left(r_{0}\right)<1 \text {, }
$$

as required. Moreover, $b_{\text {eff }}(r)<r$ near the throat and

$$
\lim _{r \rightarrow \infty} \frac{b_{\text {eff }}(r)}{r}=0 \text {, }
$$

so that the wormhole spacetime is asymptotically flat.

From the exoticity condition in Ref. [1], $b_{\text {eff }}^{\prime}\left(r_{0}\right)<1$ is equivalent to the violation of the null energy condition, requiring the need for "exotic matter." According to Equation (17) and Equation (19), however, this violation is simply a consequence of the noncommutative-geometry background.

To study the effect that the tidal forces may have on the traveler, we return to the criterion

$$
\Phi^{\prime}(r) \leq \frac{g_{\oplus}}{c^{2} \sqrt{1-b(r) / r}}
$$

mentioned in Sec. 1. At the throat of the wormhole, this condition is trivially satisfied. It is subsequently proposed in Ref. [1] that the space stations be far enough away from the throat so that $b(r) / r$ becomes negligible. The result is the requirement

$$
\Phi^{\prime} \lesssim\left(9.2 \times 10^{15} \mathrm{~m}\right)^{-1} \approx\left(10^{16} \mathrm{~m}\right)^{-1}
$$

The modified Kim-Lee model, Equation (10),

$$
\mathrm{e}^{2 \Phi}=1+R(r)+\frac{Q^{2}}{r^{2}}
$$

yields

$$
\Phi(r)=\frac{1}{2} \ln \left[1+R(r)+\frac{1}{r^{2}} \frac{2 Q^{2} \sqrt{\alpha}}{\pi}\left(\frac{1}{\sqrt{\alpha}} \tan ^{-1} \frac{r}{\sqrt{\alpha}}-\frac{r}{r^{2}+\alpha}\right)\right]
$$

and

$$
\Phi^{\prime}(r)=\frac{\frac{1}{2} R^{\prime}(r)-\frac{1}{r^{3}} \frac{2 Q^{2} \sqrt{\alpha}}{\pi}\left(\frac{1}{\sqrt{\alpha}} \tan ^{-1} \frac{r}{\sqrt{\alpha}}-\frac{r}{r^{2}+\alpha}\right)+\frac{2 Q^{2} \sqrt{\alpha}}{\pi\left(r^{2}+\alpha\right)^{2}}}{1+R(r)+\frac{1}{r^{2}} \frac{2 Q^{2} \sqrt{\alpha}}{\pi}\left(\frac{1}{\alpha} \tan ^{-1} \frac{r}{\sqrt{\alpha}}-\frac{r}{r^{2}+\alpha}\right)} .
$$

As noted earlier, $R(r)>0$ and $R^{\prime}(r)>0$, but it quickly becomes apparent that in view of Inequality (21), $R(r)$ has to be fine-tuned so that

$$
\frac{1}{2} R^{\prime}(r)-\frac{1}{r^{3}} \frac{2 Q^{2} \sqrt{\alpha}}{\pi}\left(\frac{1}{\sqrt{\alpha}} \tan ^{-1} \frac{r}{\sqrt{\alpha}}-\frac{r}{r^{2}+\alpha}\right)
$$

becomes negligibly small near the proposed location of the station. 
Remark. While the need for fine-tuning may not be desirable, it does not present a serious conceptual problem here: for large $r$, expression (24) approaches

$$
\frac{1}{2} R^{\prime}(r)-\frac{Q^{2}}{r^{3}}+\frac{2 Q^{2} \sqrt{\alpha}}{\pi r^{2}\left(r^{2}+\alpha\right)} .
$$

So $\frac{1}{2} R^{\prime} \approx Q^{2} / r^{3}$ near the station where $R(r)$ has the approximate form $-Q^{2} / r^{2}+C, C>0 ; C$ can be chosen so that the denominator in Equation (23) exceeds unity. As a result

$$
\Phi^{\prime}(r) \lesssim \frac{2 Q^{2} \sqrt{\alpha}}{\pi\left(r^{2}+\alpha\right)^{2}} .
$$

Equation (21) and Equation (23) now yield the condition

$$
\Phi^{\prime}(r) \lesssim \frac{2 Q \sqrt{\alpha}}{\pi\left(r^{2}+\alpha\right)^{2}}<\left(10^{16} \mathrm{~m}\right)^{-1} .
$$

This requirement places the following constraint on the parameter $\alpha$ :

$$
\sqrt{\alpha}<\frac{\left(r^{2}+\alpha\right)^{2} \times 10^{-16}}{\left(2 Q^{2}\right) / \pi} \mathrm{m} .
$$

According to Ref. [12], noncommutativity is not visible at presently accessible energies if $\sqrt{\alpha}<10^{-18} \mathrm{~m}$. So according to Inequality (26), the constraint on $\alpha$ is much less severe and so would in principle be observable.

\section{Conclusions}

While Morris and Thorne [1] had demonstrated that wormholes could be macroscopic structures suitable for interstellar travel, they could not avoid certain problems with traversability. In particular, the structures would have to be designed to produce low tidal forces, while the unavoidable violation of the null energy condition called for the use of "exotic matter." The latter requirement is not only difficult to meet classically, but may even be unphysical. The former was met by assuming a constant redshift function, so that $\Phi^{\prime} \equiv 0$, called the "zero-tidal-force solution".

To overcome these problems, this paper discusses the modified wormhole model due to Kim and Lee in conjunction with a noncommutative-geometry background. The violation of the null energy condition can be attributed to the latter, thereby avoiding the need for exotic matter. The presence of $Q^{2}$ in the modified Kim-Lee model allows the reduction of the tidal forces to acceptable levels without invoking the trivial zero-tidal-force assumption, again made possible by the noncommutative-geometry background. Finally, the condition on $\Phi^{\prime}$ places a constraint on the value of $\sqrt{\alpha}$, but one that is well above the limit of observability. 


\section{References}

[1] Morris, M.S. and Thorne, K.S. (1988) Wormholes in Spacetime and Their Use for Interstellar Travel: A Tool for Teaching General Relativity. American Journal of Physics, 56, 395-412. https://doi.org/10.1119/1.15620

[2] Kim, S.-W. and Lee, H. (2001) Exact Solutions of Charged Wormhole. Physical Review D, 63, Article ID: 064014. https://doi.org/10.1103/physrevd.63.064014

[3] Dehghani, M.H. and Dayyani, Z. (2009) Lorentzian Wormholes in Lovelock Gravity. Physical Revue D, 79, Article ID: 064010. https://doi.org/10.1103/PhysRevD.79.064010

[4] Zangeneh, M.K., Lobo, F.S.N. and Dehghani, M.H. (2015) Traversable Wormholes Satisfying the Weak Energy Condition in Third-Order Lovelock Gravity. Physical Review D, 92, Article ID: 124049. https://doi.org/10.1103/physrevd.92.124049

[5] Mehdizadeh, M.R., Zangeneh, M.K. and Lobo, F.S.N. (2015) Higher-Dimensional Thin-Shell Wormholes in Third-Order Lovelock Gravity. Physical Review D, 92, Article ID: 044022. https://doi.org/10.1103/physrevd.92.044022

[6] Mehdizadeh, M.R., Zangeneh, M.K. and Lobo, F.S.N. (2015) Einstein-Gauss-Bonnet Traversable Wormholes Satisfying the Weak Energy Condition. Physical Review $D$. 91, Article ID: 084004. https://doi.org/10.1103/physrevd.91.084004

[7] Zangeneh, M.K., Lobo, F.S.N. and Riazi, N. (2014) Higher-Dimensional Evolving Wormholes Satisfying the Null Energy Condition. Physical Review D, 90, Article ID: 024072. https://doi.org/10.1103/physrevd.90.024072

[8] Kuhfittig, P.K.F. (2015) Macroscopic Traversable Wormholes with Zero Tidal Forces Inspired by Noncommutative Geometry. International Journal of Modern Physics D, 24, Article ID: 1550023. https://doi.org/10.1142/s0218271815500236

[9] Witten, E. (1996) Bound States of Strings and p-Branes. Nuclear Physics B, 460, 335-350. https://doi.org/10.1016/0550-3213(95)00610-9

[10] Seiberg, N. and Witten, E. (1999) String Theory and Noncommutative Geometry. Journal of High Energy Physics, 9909, Article ID: 032. https://doi.org/10.1088/1126-6708/1999/09/032

[11] Smailagic, A. and Spallucci, E. (2003) Feynman Path Integral on the Non-Commutative Plane. Journal of Physics A, 36, L467-L471.

https://doi.org/10.1088/0305-4470/36/33/101

[12] Nicolini, P., Smailagic, A. and Spallucci, E. (2006) Noncommutative Geometry Inspired Schwarzschild Black Hole. Physics Letters B, 632, 547-551.

https://doi.org/10.1016/j.physletb.2005.11.004

[13] Rinaldi, M. (2011) A New Approach to Non-Commutative Inflation. Classical and Quantum Gravity, 28, Article ID: 105022. https://doi.org/10.1088/0264-9381/28/10/105022

[14] Garattini, R. and Lobo, F.S.N. (2009) Self-Sustained Traversable Wormholes in Noncommutative Geometry. Physics Letters B, 71, 146-152.

https://doi.org/10.1016/j.physletb.2008.11.064

[15] Lobo, F.S.N. and Garattini, R. (2013) Linearized Stability Analysis of Gravastars in Noncommutative Geometry. Journal of High Energy Physics, 1312, Article ID: 065. https://doi.org/10.1007/jhep12(2013)065

[16] Kuhfittig, P.K.F. (2016) Gravitational Lensing of Wormholes in Noncommutative Geometry. Scientific Voyage, 2, 1-8.

[17] Rahaman, F., Kuhfittig, P.K.F., Ray, S. and Islam, S. (2012) Searching for Higher Dimensional Wormholes with Noncommutative Geometry. Physical Review D, 86, 
Article ID: 106101. https://doi.org/10.1103/physrevd.86.106010

[18] Kuhfittig, P.K.F. (2013) Macroscopic Wormholes in Noncommutative Geometry. International Journal of Pure and Applied Mathematics, 89, 401-408.

https://doi.org/10.12732/ijpam.v89i3.11

[19] Liang, J. and Liu, B. (2012) Thermodynamics of Noncommutative Geometry Inspired BTZ Black Holes Based on Lorentzian Smeared Mass Distribution. Europhysics Letters, 100, Article ID: 30001. https://doi.org/10.1209/0295-5075/100/30001

[20] Nozari, K. and Mehdipour, S.H. (2008) Hawking Radiation as Quantum Tunneling for a Noncommutative Schwarzschild Black Hole. Classical and Quantum Gravity, 25, Article ID: 175015. https://doi.org/10.1088/0264-9381/25/17/175015

[21] Sushkov, S.V. (2005) Wormholes Supported by a Phantom Energy. Physical Review $D$, 71, Article ID: 043520. https://doi.org/10.1103/physrevd.71.043520

[22] Kuhfittig, P.K.F. (2011) On the Feasibility of Charged Wormholes. Central European Journal of Physics, 9, 1144-1150. https://doi.org/10.2478/s11534-011-0043-2

[23] Kuhfittig, P.K.F. (2016) The Effect of Conformal Symmetry on Charged Wormholes. Journal of Applied Mathematics and Physics, 4, 2117-2125.

https://doi.org/10.4236/jamp.2016.412209

Submit or recommend next manuscript to SCIRP and we will provide best service for you:

Accepting pre-submission inquiries through Email, Facebook, LinkedIn, Twitter, etc. A wide selection of journals (inclusive of 9 subjects, more than 200 journals)

Providing 24-hour high-quality service

User-friendly online submission system

Fair and swift peer-review system

Efficient typesetting and proofreading procedure

Display of the result of downloads and visits, as well as the number of cited articles

Maximum dissemination of your research work

Submit your manuscript at: http://papersubmission.scirp.org/

Or contact jamp@scirp.org 